\title{
Beyond "Biosignatures": Importance of Applying Abiotic / Prebiotic Chemistry to the Search for Extraterrestrial Life
}

Lead Author: Laura M. Barge

NASA Jet Propulsion Laboratory, California Institute of Technology

818-393-8209; Laura.m.barge@jpl.nasa.gov

Co-Authors:

Laura Rodriguez (NASA JPL), Jessica M. Weber (NASA JPL), Bethany Theiling (NASA GSFC)

\section{Co-Signers:}

Pin Chen (NASA JPL), Scott Perl (NASA JPL), Bryana Henderson (NASA JPL), Julie Castillo (NASA JPL), Nicolle Zellner (Albion College), Morgan Cable (NASA JPL), Jason Dworkin (NASA GSFC), Robert Hodyss (NASA JPL), Edith Fayolle (NASA JPL), Pat Beauchamp (NASA JPL), Aaron Engelhart (University of Minnesota), Jennifer G. Blank (NASA Ames / Blue Marble Space Institute of Science), Pablo Sobron (Impossible Sensing), John-Paul Jones (NASA JPL), Keith Chin (NASA JPL), Sanjoy Som (Blue Marble Space Institute of Science), Alexis Templeton (University of Colorado), Yuk Yung (Caltech), Tiffany Kataria (NASA JPL), Aaron Goldman (Oberlin College), Tony Freeman (NASA JPL), Aaron Burton (NASA JSC), Matt Pasek (USF), Abigail Fraeman (NASA JPL), Laura Kerber (NASA JPL), Britney Schmidt (Georgia Tech), Mohit Melwani Daswani (NASA JPL), Bethany Ehlmann (Caltech), Milena Popovic (Blue Marble Space Institute of Science)

Acknowledgement: This research was carried out at the Jet Propulsion Laboratory, California Institute of Technology, under a contract with the National Aeronautics and Space Administration (80NM0018D0004). @ 2020, All rights reserved. 


\section{Key points:}

- The boundary between abiotic and biotic chemistry could vary significantly, depending on the geological environment. It is possible that the same chemical signature observed on two different worlds could be biological in one and have an abiotic origin in another, depending on the geobiological state of those worlds.

- The burden of proof for classifying a chemical signature as biological is higher for other worlds than for Earth. Indeed, many of the chemical signatures that we identify as "biological," and would certainly be evidence for life within an Earth sample, cannot be used unambiguously as indicators of life elsewhere.

- Abiotic vs. biotic is not a dichotomy, but a spectrum. While samples from Earth represent an excellent "end-member" for what biological systems might be capable of, Earth field samples cannot represent the "abiotic end-member" of this spectrum, because biology has suppressed prebiotic / abiotic chemistry on this planet.

- To more accurately interpret whether an organic signature from another world is a biosignature, we need to gain a more complete understanding of abiotic chemical possibilities and create a library of analog samples for mission instruments to assess. Due to prevalence of Earth life, these will need to be laboratory-generated samples, rather than samples from the field, compared to samples analyzed in situ or collected from a variety of planetary surfaces.

\section{Introduction:}

The purpose of this white paper is to urge NASA to continue to invest in understanding the boundaries between abiotic and biotic systems, and to demonstrate how prebiotic and origin of life chemistry is an essential part of searching for life elsewhere as we move into the next decade of planetary missions. Many NASA missions seek to find signs of biology on other worlds. This not only influences instrument and mission design, but also affects the types of Research and Development (R\&D) projects that are funded to provide analytical standards, field / lab analogs, interpretation frameworks for possible biosignatures, and instrumentation capable of identifying biosignatures. The general approach for finding life elsewhere is to try to find commonalities between all forms of life as we observe it, and then try to find phenomena that we do not observe to happen abiotically. These phenomena are "biosignatures" that form the framework for mission science goals and instrument development. However, an area that is often overlooked in planetary science / astrobiology is the importance of abiotic organic chemistry beyond that observed in Earth geological sites.

On Earth today, biology has permeated all Earth-surface and near-surface environments. Therefore, it is reasonable to assume that any naturally occurring highly complex organic systems and/or systems capable of energy conversion and transfer in a field sample likely result from life or at least had past biological influence. Modern biological metabolism is a highly efficient process that is capable of kinetically / thermodynamically dominating a variety of redox niches [1], selectively synthesizing complex functional organic molecules, and does so in a wide 
variety of "habitable" environments. Consequently, abiotic organic chemistry in most terrestrial environments is fairly simple, producing small organic molecules (e.g., amino acids or carbon reduction in hydrothermal systems [2]) or disorganized "tars" which encompass a wide range of chemical components in random arrangements and are often insoluble in solution. This is not because abiotic organic chemistry is only capable of producing such simple compounds! It is because Earth's biosphere currently dominates, and suppresses / obscures the majority of abiotic chemistry that would otherwise be possible. Therefore, when assessing organic signatures detected by a mission on another world where we cannot be certain whether life has even emerged, it is important to take into account the true limits of abiotic organic chemistry, beyond just what we observe in terrestrial field samples.

Going forward, the following questions should be considered when discussing biosignatures:

- What is the maximum complexity (defined as a factor of molecular size, number of heteroatoms (i.e. other than $\mathrm{C}$ or $\mathrm{H}$ ) and degree of organization (e.g., chirality or repeating units)) that abiotic chemistry in a geological environment is capable of?

- And likewise, what types of organic signatures on another planet could be produced abiotically even though we are unable to differentiate this abiotic process on Earth?

These questions can only be explored in the laboratory, where the influence of biology can be controlled or eliminated, to correctly interpret mission data of organics in situ or in returned samples. Many of the chemical phenomena that we associate with life can also transpire in non-living systems when life is excluded. Laboratory studies simulating prebiotic conditions have demonstrated the following synthesis, for example: amino acid and nucleotide synthesis; formation of amino acid / hydroxy acid / nucleotide polymers; formation of oligomers capable of information content and transfer; formation of lipid membranes; the encapsulation of both reactions and polymers within said membranes, and how replication of the encapsulated polymers triggers replication of the lipid membrane containing the encapsulated polymer; and enzyme-like metal catalysts that can drive whole networks of proto-metabolic reactions [3]. In sum, it is possible to create extremely complex, nearly life-like organic chemical systems completely abiotically, as decades of origin of life research have demonstrated. In order for life to exist on this planet, extremely complex abiotic chemistry must have emerged first, and at some point that abiotic chemistry must have been nearly indistinguishable from the actual life it was transitioning into. Prebiotic chemistry focuses on re-creating steps that could have led to life on Earth, but even that is only a subset of the possible abiotic organic chemistry that could take place in a planetary environment: early Earth contained many reactive environments in addition to those that actually drove the origin of life; other planets contain still more unique sets of conditions. In this regard, astrobiologists should also take note of synthetic chemistry literature to stay abreast of the actual state of the art in abiotic organic synthesis. For example, chemists can now abiotically synthesize chains of hundreds of amino acids to make artificial proteins [4], and 100-unit-long sugar polymers [5]. It is certainly not the case that "abiotic organic chemistry" is limited to Fischer-Tropsch reactions or organic distributions from meteorites.

Given this, the questions remain:

- How would mission instruments that are designed to search for life interpret complex prebiotic/abiotic chemistry such as that which could have formed on the Hadean Earth or from a modern abiotic organic synthesis?

- Would there be a mission-instrument-detectable difference between: 
- Tars and a large, complex polymer?

$\circ$ A living, replicating cell and an abiotic protocell containing organic polymers that trigger vesicle replication?

Such questions merit further investigation.

\section{Interpreting organic detections from missions:}

Given that abiotic organic chemistry can be extremely complex, we should not rely on Earth field samples and meteorites as representative endmembers of such chemistry: biology inhibits complex abiotic chemistry on Earth, and the geochemical conditions meteorites are exposed to are limited compared to that of a planetary body which may host a myriad of environments. Thus, the question remains of where then to place the boundary between "abiotic" and "biotic" in a mission context. The difficulty for life detection is that this is not a constant answer, and the boundary between abiotic organic chemistry and a biosignature depends on the planet's overall geobiological state. Consider Earth at various points throughout its history (Figure 1): 1) Before the emergence of life, 2) Just after the emergence of the Last Universal Common Ancestor (LUCA) but before the diversification and spread of life, 3) 541 million years ago upon the advent of the 'Cambrian Explosion'; a flurry of biological diversification took place across marine environments globally, as evidenced by a global fossil record [6]. In all these cases, a planetary mission might be able to detect "complex" organic systems. However, evaluating whether identified organics were generated by life would depend on the degree to which life has

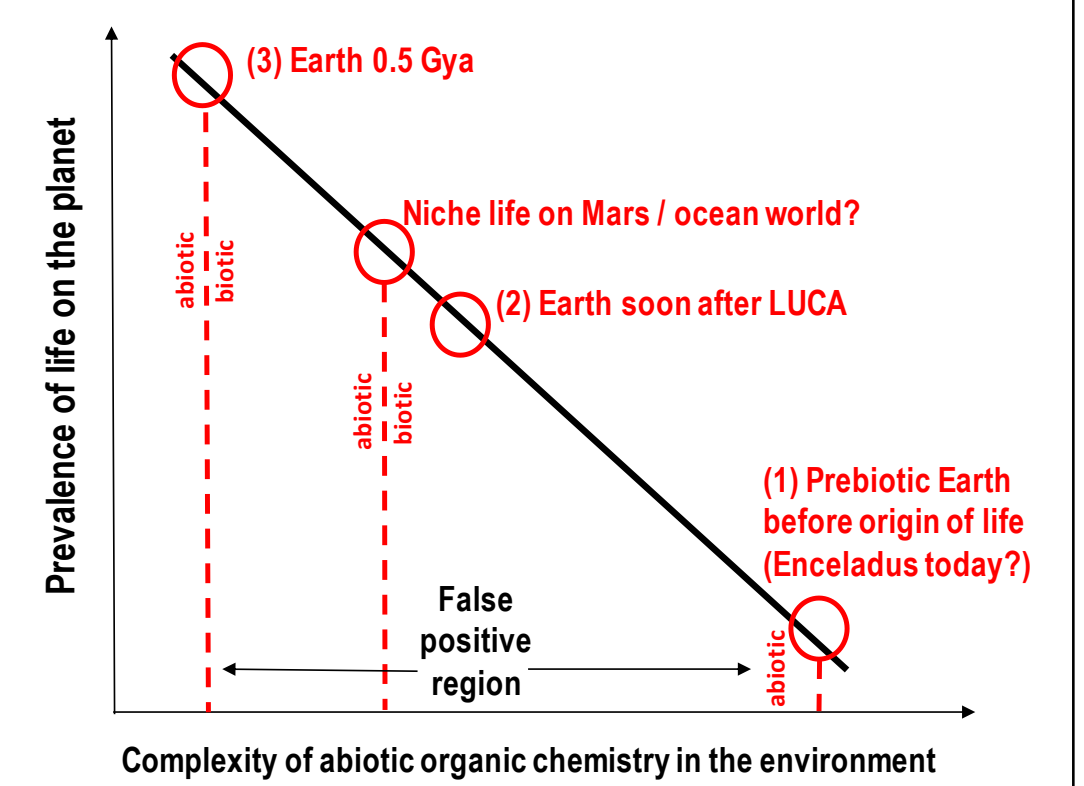

Figure 1: The boundary between "abiotic" and "biotic" organic chemistry can shift depending on the geobiological state of the planet (graph is not to scale and is not necessarily a linear relationship). To determine whether an organic detection from a mission is a biosignature, we need a more complete understanding of where that boundary lies for each world or environment. spread and suppressed abiotic organic chemistry. In scenario (1) (Figure 1), since there is no life, any complex organic chemistry detected (e.g., a vesicle containing proto-RNA and proto-metabolism) would be abiotic. In scenario (2), the most complex organic chemistry (e.g., LUCA, a cell with genetic material and metabolism) would be biological, but since biology was not widespread, there might also be complex abiotic organic chemistry in other samples (e.g., oligomer synthesis, proto-metabolism). In scenario (3), any sample containing e.g., nucleotide polymers or 100-mer amino acid oligomers is probably a biosignature, since at this point life had spread over the whole planet and would 
suppress the abiotic formation of these signatures (Figure 1). Similar to the "dynamic habitability" concept [7], as planetary conditions evolve, the definition of a biosignature on a planet should also evolve.

For other planets, there could be any number of possibilities in this regard. Figure 1 shows a proposed relation between the prevalence of life on a planet and the complexity of abiotic organic chemistry that might be found in the environment. The three scenarios described above for Earth are plotted on this graph, and also hypothetical scenarios of interest for Mars or ocean worlds. For example, organics have been detected on Enceladus [8], and some researchers have suggested that Enceladus may harbor prebiotic chemistry today [9]; if this is true, then perhaps Enceladus is producing complex abiotic organic chemistry (as Earth did before the origin of life) and comparing its organic detections to Earth biological systems is not appropriate. As another example, if there is / was life on Mars, perhaps it is / was not as widespread and dominant as life on Earth and is / was confined to isolated niches [10]. If that is the case, then perhaps putative Martian life has not suppressed abiotic / prebiotic organic chemistry to the extent that Earth life has, and the threshold for 'biotic' organics for Mars would actually be shifted farther to the right on Figure 1's X-axis. The main point is that: for any world that is different from Earth in terms of the prevalence of life (which may be all of the planets we have visited so far), the threshold for identifying a particular organic molecule / process as biological would be different from what we would assume based on Earth field samples. In this schematic, Earth lies to the high end of planetary biological influence, meaning there is a "false positive" region: if any organic material of chemical complexity approaching that expected for Earth life were detected on another world with no life (or less prevalent life), it could give a false positive for life detection if we compare it to an Earth-centric abiotic / biotic boundary instead of the appropriate abiotic chemical analogs for other worlds. In general, it must be assumed that an organic signature is not life until proven otherwise by multiple techniques, since there are so many pathways to making complex organic signatures abiotically, and these abiotic pathways could be even more prevalent on other worlds than they are on Earth.

\section{Determining appropriate abiotic organic analogs for biosignature studies:}

The fields of prebiotic chemistry and synthetic chemistry provide many interesting examples of what might be possible for non-biological organic systems. Also, laboratory experiments and numerical models can inform one another to increase the range of environmental parameters under which abiotic chemistry can be studied, even beyond conditions that are readily attainable in the laboratory. However, it is also necessary to keep geological context in mind, since in the laboratory we can create chemical conditions that are unlikely to occur in a planetary environment. There is such a variety of mechanisms, catalysts, and conditions that can drive the formation of complex organic molecules and reaction networks; therefore, variable environments on planets could contain numerous combinations of 'favorable' conditions. In order to better define appropriate abiotic organic chemistry analogs for planetary mission studies, we would suggest two paths forward.

- First, it would be desirable to build a stronger relationship between astrobiologists and synthetic chemists, to not just analyze specific molecules produced for industrial purposes, but to consider how any of the general mechanisms, catalysts, and/or pathways utilized by state of the art chemical synthesis might be applicable to abiotic geological settings. 
- Second, we should increase research into prebiotic chemical scenarios. This research is not solely to discover how life emerged on Earth, but to better explore the range of "dead end" possibilities that could have resulted in a persistent complex abiotic chemical system that, for whatever reason, did not result in an origin of life. It will also enable us to explore the range of alternative origins and/or outcomes from organic geochemical systems that could give rise to complex systems unlike what we see on Earth [11]. Together, these approaches would help to increase knowledge of how to interpret organic chemical signatures from missions, and help to move away from our Earth-centric definitions of what constitutes a biosignature.

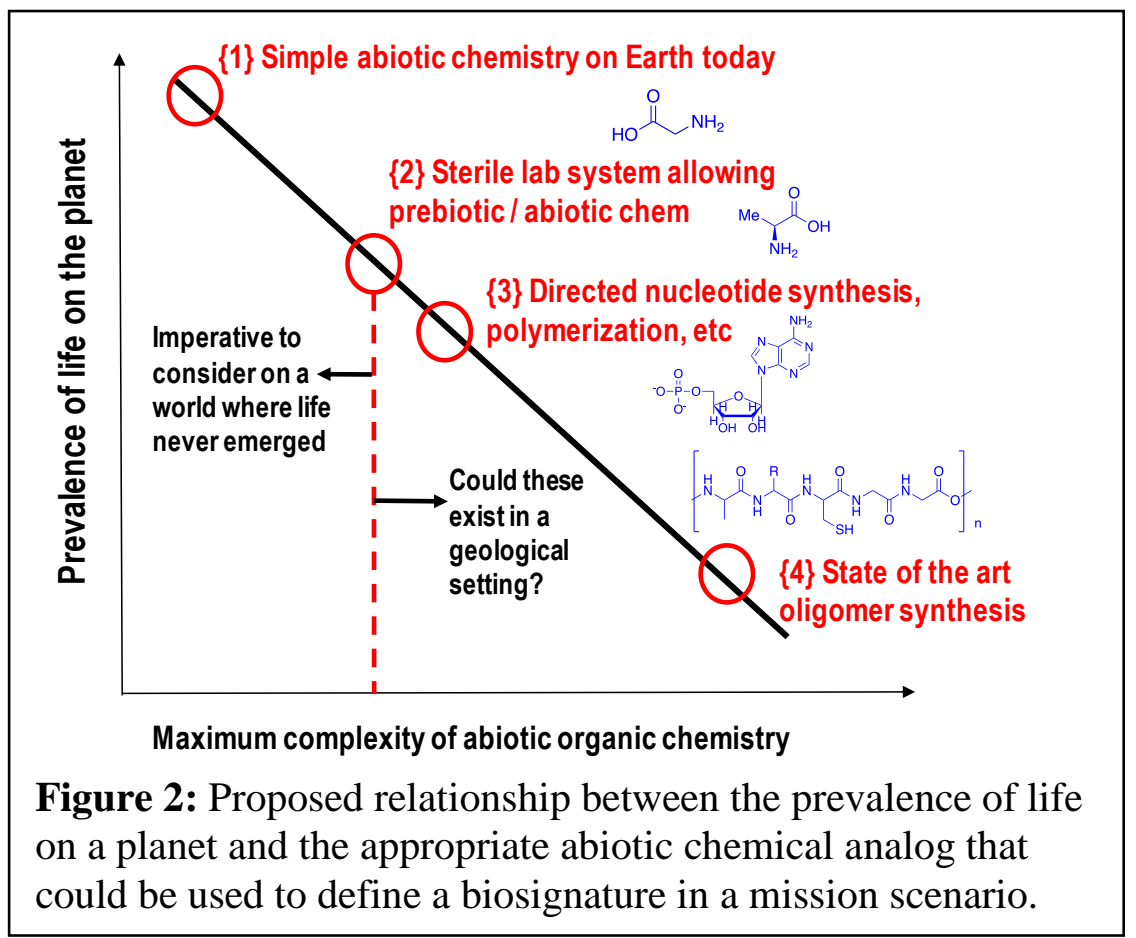

Figure 2 shows a similar schematic to Figure 1, but this time focusing on the relationship between the supposed prevalence of life on a planet, and the practical appropriate abiotic chemical analog(s) that a mission or instrument could use to help define the biotic / abiotic boundary. For a sample from Earth, it would be appropriate to consider only comparisons to known biological systems and assume that abiotic chemistry is simple $\{1\}$. Since we do not know if life emerged anywhere

else, the baseline for an abiotic analog for any other planet should be a laboratory system that was kept strictly free of life and abiotic organic processes allowed to flourish $\{2\}$. This could be a prebiotic chemistry experiment targeted at Earth's origin of life, or an experiment with organics more targeted toward that planet's current environment, e.g., abiotic reactions of organics with Mars-relevant minerals and gradients. Depending on the outcome of conversations between astrobiologists and synthetic chemists, other more sophisticated examples could also be relevant, e.g., conditions that lead to high yield abiotic syntheses of biomolecules $\{3\}$ or even the state of the art in making long, complex oligomers abiotically $\{4\}$.

\section{Recommendations to NASA:}

In summary, we suggest the following recommendations going forward:

1) NASA should continue to support laboratory, field, and modeling research related to prebiotic / origin of life systems, both targeted for understanding processes that could have led to Earth's biosphere, but also for processes that could lead to emergence of life elsewhere under different conditions (and including emergent complexity systems that could be prebiotic but may have resulted in "dead ends"). 
2) Any interpretation of biosignatures on another planet should take into account that the boundary between biological and non-biological organic chemistry in an environmental sample is determined by the planet's geobiological state; this boundary should change with time throughout a planet's history (similar to its habitability), and would not be a constant parameter defined by samples from Earth field sites.

3) Life detection oriented missions should be prioritized by this Decadal Survey; and in doing so we should create a new standard of "abiotic complex organic chemistry" analog samples, to serve as must-have test cases for in situ planetary instruments that are designed to look for life. (For example, an instrument designed to detect biological DNA would also want to analyze a synthetic nucleotide oligomer produced abiotically, not just compare to 'meteorite distributions'.)

4) Instrument requirements should continue to be defined by detection limits of molecules / conditions of interest, but keep in mind that "life detection" is a complex process that would be specific to that planetary environment, and is not satisfied by observation of any specific molecule or organic distribution unless the abiotic possibilities are well understood.

5) R\&D efforts should also invest in experimental studies to determine abiotic organic chemical possibilities on worlds of interest, by testing organic reactions in these environments (without life) and producing abiotic samples for instruments to compare to. This could be accomplished under existing programs such as Exobiology that study biosignatures, but with increased investment in research that focuses on abiotic / prebiotic chemistry in other planetary environments besides Earth.

6) NASA should invest in developing a sample collection of complex abiotic organic standards for the astrobiology community, that draws from not just Earth field samples and Earth-based origin of life experiments, but also the chemical synthesis world, and experiments targeted at simulating other planets and scenarios that are not Earth-like. This could be accompanied by a database that compiles reactions that have been run as a reference to check to see if a given molecule or organic signature has already been made abiotically / prebiotically. This collection should be improved / added to continuously as our capabilities and understanding of abiotic mechanisms advances. It should be standard protocol for organic detection mission instruments to analyze these abiotic comparisons as well as biological examples.

\section{References:}

[1] Nealson, K. (2003) Nat. Biotechnol. 21, 243-244.

[2] Ménez, B. et al. (2018) Nature 564, 59-63.

[3] Barge L. M. et al. (2019) PNAS, 116 (11) 4828-4833; Chandru K. et al. (2018) Nat. Comm. Chem. 1, 30; Da Silva, L. et al. (2015) JME 80, 86-97; Forsythe, J.G., et al. (2017) PNAS 114, E7652-E7659; Gibard, C., et al. (2018) Nat. Chem. 10, 212-217; Huber, C., Wächtershäuser, G. (2003) Tetrahedron Letters 44(8): 1695-1697; Jia, T.Z., et al. (2019) PNAS 116 (32) 1583015835; Jordan, S.F., et al. (2019) Nat. Ecol. Evol. 3, 1705-1714; Kim, H.-J., Benner, S. A. (2018) Chem. Eur. J. 24, 581-584; Kurihara, K., et al. (2011) Nat. Chem. 3: 775-781; Maurer, S., Nguyen, G. (2016) OLEB 46(2-3):215-22; Muchowska, K. et al. (2019) Nature 569: 104-107; Olasagasti, F., Rajamani, S. (2019) Life 9(4): 83; Rodriguez, L.E., et al. (2019) Scientific Reports 9, 9281; Wang, W., et al. (2011) Astrobiology 11, 471-476. 
[4] Hartrampf N. et al. (2020) Science 368, 6494, 980-987.

[5] Joseph A.A. et al. (2020) J. Am. Chem. Soc. 142, 19, 8561-8564.

[6] Marshall C. R. (2006) Ann. Rev. Earth Plan. Sci. 34:355-384.

[7] National Academies of Sciences, Engineering, and Medicine 2019. An Astrobiology Strategy

for the Search for Life in the Universe. Washington, DC: The National Academies Press.

https://doi.org/10.17226/25252.

[8] Postberg, F., et al. (2018) Nature 558, 564-568.

[9] Kahana A., et al. (2019) Astrobiology 19, 10, 1263-1278.

[10] Michalski, J.R., et al. (2017). Nature Geoscience 11: 1-26.

[11] Scharf C., et al. (2015) Astrobiology 15(12):1031-1042. 